\title{
A Novel $L D L R$ variant in a Ukrainian Patient: A Case Report and Overview of the Disease-Causing LDLR Variants Associated to Familial Hypercholesterolemia
}

\section{Simona Moffa}

Fondazione Policlinico Universitario Agostino Gemelli IRCCS

\section{Maria Elisabetta Onori}

Fondazione Policlinico Universitario Agostino Gemelli IRCCS

\section{Elisa De Paolis}

Fondazione Policlinico Universitario Agostino Gemelli IRCCS

Claudio Ricciardi Tenore

Fondazione Policlinico Universitario Agostino Gemelli IRCCS

\section{Alessia Perrucci}

Fondazione Policlinico Universitario Agostino Gemelli IRCCS

\section{Alfredo Pontecorvi}

Fondazione Policlinico Universitario Agostino Gemelli IRCCS

\section{Andrea Giaccari}

Fondazione Policlinico Universitario Agostino Gemelli IRCCS

\section{Andrea Urbani}

Fondazione Policlinico Universitario Agostino Gemelli IRCCS

Angelo Minucci ( $\square$ angelo.minucci@policlinicogemelli.it )

Teaching and Research Hospital "Agostino Gemelli" Foundation, Rome, Italy https://orcid.org/00000002-0833-4334

\section{Short Report}

Keywords: Familial hypercholesterolemia, Next Generation Sequencing, LDL-cholesterol, FH-Devyser Kit, novel LDLR variant, Ukrainian population

Posted Date: September 7th, 2021

DOl: https://doi.org/10.21203/rs.3.rs-850927/v1

License: (c) (1) This work is licensed under a Creative Commons Attribution 4.0 International License. Read Full License 
Page 2/13 


\section{Abstract}

Familial hypercholesterolemia $(\mathrm{FH})$ is characterized by high low-density lipoprotein-cholesterol levels and it is primarily caused by pathogenic/likely pathogenic variants (P/LPVs) in $L D L R, A P O B$ or PCSK9 genes.

Next Generation Sequencing (NGS) technology allows the evaluation of more genes simultaneously, rising the diagnostic throughput of genomics laboratories.

We report a Ukrainian 37-year-old woman hypercholesterolemic since 2010. Despite a suggestive family history, FH was suspected only when the patient referred to the Endocrine and Metabolic Diseases Center of the Fondazione Policlinico Universitario A. Gemelli IRCCS in Rome. After specialist advice, genetic testing was offered to the patient at our Molecular and Genomic Diagnostics Unit.

A targeted NGS-based pipeline highlighted a novel out-of-frame deletion in the $L D L R$ gene. This variant has a clear deleterious effect on the LDLR protein and it can be classified as PV.

The ideal model of care for $\mathrm{FH}$ is an evidence-based system aimed to provide the highest-quality health services to all FH patients. In fact, this study reports that an integrated care pathway adopted in our hospital for $\mathrm{FH}$ patients led successfully to the discovery of a novel $L D L R \mathrm{PV}$ in a Ukrainian patient. The finding of this $L D L R$ variant allowed the clinical FH diagnosis in this patient and in her family, expanding the knowledge of FH-related genetic variants in the Ukrainian population.

\section{Introduction}

Familial hypercholesterolemia $(\mathrm{FH})$ is a common hereditary disorder characterized by high levels of lowdensity lipoprotein cholesterol (LDL-C). Recent studies have shown that FH is underdiagnosed in many parts of the world and it has been estimated that less than $10 \%$ of $\mathrm{FH}$ patients worldwide have been diagnosed with the condition [1, 2].

FH can be diagnosed using different clinical diagnostic criteria, after ruling out secondary causes of hypercholesterolemia. However, in case of younger individuals or in those with a milder phenotype, the clinical diagnosis of FH could be difficult due to polygenic causes of raised LDL-C levels.

Clinical diagnostic criteria are mainly based on lipid levels and on combinations of physical signs and personal or family history of hyperlipidemia and/or premature coronary artery disease [3]. Typically, diagnosis is made using one of the Dutch Lipid Clinic Network (DCLN) [4], Simon Broome Register [5] or Make Early Diagnosis to Prevent Early Death [6] criteria, each one requires a different set of patient data. Although these diagnostic criteria have been extensively used, genetic testing is the preferred method, because it allows an unequivocal $\mathrm{FH}$ diagnosis and provides also information for family cascade screening tests [7].

In 2018, the Familial Hypercholesterolemia Foundation promoted an assessment of the usefulness of genetic testing for $\mathrm{FH}$ curated by international experts. They recommended the genetic testing for $\mathrm{FH}$ as 
standard of disease management in people with definitive or probable $\mathrm{FH}$ and for their at-risk relatives [8].

Genetic testing should include at least 3 main genes: the LDL-receptor $(L D L R)$ gene, accounting for over $90 \%$ of the cases, the apolipoprotein B-100 (APOB) gene, accounting for 5 to $10 \%$ of the cases, and the proprotein convertase subtilisin/kexin type 9 (PCSK9) gene accounting for up to $3 \%$ of the cases [9]. In addition, other genes as APOE, LDLR adaptor protein 1 (LDLRAP1) and signal-transducing adaptor family member 1 (STAP1) genes should be considered as rare causes of autosomal dominant/recessive FH forms [10].

For these reasons, the genetic evaluation, which includes at least the abovementioned genes, is mandatory in case of patients suggestive for the FH and Next Generation Sequencing (NGS), now widely used in the clinical setting, offers an efficient and comprehensive molecular approach for these patients. However, the diagnostic performance of any genetic test can vary greatly depending on the population studied and the NGS platform used. In this context, the mutation detection rate is also higher when genetic testing is performed in patients with a clear suspicion of $\mathrm{FH}$ [9].

The spectrum of pathogenic/likely pathogenic variants (P/LPVs) associated to $\mathrm{FH}$ is continuously updated because new variants are constantly annotated, also thanks to the increased and widespread use of NGS in the genomics laboratories [11].

In this study, we report a Ukrainian patient with a pesonal history of hypercholesterolemia. Despite this suggestive evidence, a specialist consultation, aimed to investigate the possible FH status, was never proposed to the patient. Referred to Center for Endocrine and Metabolic Diseases of our hospital, the patient was addressed to the appropriate genetic test performed via a targeted NGS (tNGS) multi-panel approach [11]. The patient signed written informed consent before genetic test.

The molecular evaluation highlighted a previously unreported $L D L R$ frame-shift variant, predicted to be a deleterious variant.

In our opinion, the case here described could be newsworthy considering that: 1) an integrated care pathway adopted in our hospital for $\mathrm{FH}$ patients management led to a definitive clinical classification of the FH status, discovering a novel $L D L R \mathrm{PV} ; 2$ ) the new variant added information about molecular basis of $\mathrm{FH}$ in the Ukrainian population, in which data concerning the prevalence and distribution of $\mathrm{FH}$ derive only from two projects: the ScreenPro FH Project and the European Atherosclerosis Society FH Studies Collaboration (EAS FHSC) $[12,13]$ and 3 ) the new variant allowed to broaden the spectrum of $L D L R$ variants. In this context, a summary of all P/LPVs currently reported in the $L D L R$ gene is also provided in this study.

Finally, we should also carefully speculate that the peculiar $L D L R$ variant reported in this study could be a founder variant in the Ukrainian population. Further studies are needed to confirm or deny this hypothesis. 


\section{Case Presentation}

The patient is a 37-year-old woman, born in Lugansk (Ukraine), who referred to our Center in December 2018. Her medical history began in 2010, when she was diagnosed with hypercolesterolemic. Since then, she has been treated with several statins, always interrupted due to the onset of intolerable muscle pain. The patient came to our attention to access alternative lipid-lowering therapies. Until then, no one suspected FH. Her lipid profile at the moment of first diagnosis and later, in July 2018, with no lipidlowering therapy, is reported in table 1 , which was strongly suggestive for $\mathrm{FH}$. Therefore, we investigated her family history, discovering her mother's hypercholesterolemic without any cardiovascular events; while her maternal uncle and grandfather died at the age of 33 and 38, respectively, for acute myocardial infarction. On physical examination, she showed no tendon xanthoma or corneal arcus. The patient had no other cardio-vascular risk factors; she had a body mass index of $23 \mathrm{~kg} / \mathrm{m} 2$ and no previous history of coronary heart disease; she was normotensive, non-diabetic and did not smoke. Based on DCLN diagnostic criteria for $\mathrm{FH}$ [4], the score reached by our patient was 10 , standing for a definite diagnosis of $\mathrm{FH}$; for this reason and according to EAS/European Society of Cardiology (ESC) guidelines [14], we performed an ad hoc genetic evaluation.

Considering the very high values of LDL-C after the first visit, she was prescribed with rosuvastatin $40 \mathrm{mg}$ and ezetimibe $10 \mathrm{mg}$. Despite this maximal therapy, her LDL-C, although significantly reduced, was not yet at target level; for this reason, she started in June 2019 therapy with anti-PCSK9 antibodies that is still ongoing. As reported in table 1, her lipid profile is currently at target level, according to the ESC/EAS guidelines of 2016 and 2019 [14].

Genomic DNA was isolated from peripheral blood using an automated device (MagCore HF16 Plus, Diatech Lab Line, Jesi, Italy). Targeted sequencing of six genes (LDLR, APOB, PCSK9, APOE, LDLRAP1 and STAP1) was performed using a NGS-based approach (Devyser FH, Devyser, Stockholm, Sweden). Illumina MiSeq platform (Illumina, San Diego, CA, USA) and Amplicon Suite software (Smart seq, Novara, Italy) were used for sequencing and data analysis, respectively [11].

NGS did not reveal any known P/LPVs and bioinformatics prediction was not indicative for the presence of copy number alterations (CNAs) in the investigated genes. However, a 14 bp deletion c.171_184del, p. (Glu58ValfsTer67) (coverage: 376/857X, variant allele frequency (VAF): 44\%) in the LDLR gene was highlighted (Fig. 1). The nomenclature of the variant is based on the $L D L R$ sequence (NCBI Reference Sequence: NM_000527.5; GRCh37), according to the recommendations of the Human Genome Variation Society (https://www.hgvs.org).

This variant was considered nove/ since it was not present in in the main variant databases as: Clin Var (https://www.ncbi.nlm.nih.gov/clinvar/), Leiden Open-source Variation Database(LOVD) (https://databases.lovd.nl/shared/genes/LDLR), Exome Aggregation Consortium (ExAC) (http://exac.broadinstitute.org), 1000Genomes (http://www.internationalgenome.org/1000-genomesbrowsers/) and Varsome (https://varsome.com/) (last access June 2021). In addition, we did not identify 
this variant in our cohort of about $350 \mathrm{FH}$ patients routinely analyzed with the same tNGS approach adopted in this study.

Unfortunately, it was not possible to screen the variant in the family members.

Confirmation of NGS data via Sanger-based sequencing and identification of the c.171_184del variant by alternative approaches

In order to confirm the presence of the LDLR C.171_184de/ variant highlighted by NGS, a targeted Sanger sequencing was used. Primers able to amplify and sequence the exon 2 of the $L D L R$ gene were designed by using Primers 3 software (http://primer3.ut.ee/). Their sequences were: 5'- GATTCTGGCGTTGAGAGAC - 3' (forward) and 5'- CGAGACCCTGTCTCTATTAC - 3' (reverse) (Fig. 2).

Considering the peculiarity of the detected variant (a 14 nucleotides deletion), alternative molecular approaches were used In particular, we performed an electrophoresis on $4 \%$ agarose gel and a HighResolution melting Analysis (HRMA) (Fig. 2), evaluating the different size and melting profiles of the PCR products, respectively $[15,16]$.

Both alternative approaches successfully identified the c.171_184del variant from the wt allele.

\section{Overview of the P/LPVs in the LDLR gene}

The new variant reported in this study broadens the spectrum of P/LPVs in the $L D L R$ gene. To date, more than $2030 L D L R$ variants have been reported in the FH LOVD database, updated in June 2021. FH is primarily associated to loss-of-function point variants spread within the $L D L R$ gene (>95\%) [3]. We collected all previously reported P/LPVs in the LDLR gene, providing a classification based on variant type (nonsense, missense, indels, splicing, synonymous variants and CNAs).

Overall, a total of 1452 unique P/LPVs accounts in the coding regions and in the exon/intron junction regions. Figure 3 depicted the variants distribution as: 127 nonsense, 644 missense, 418 indels, 111 CNAs, 132 splicing and 20 synonymous variants.

Finally, Fig. 4 shows the linear map of the $L D L R$ gene together with the distribution of the genetic variants (https://pecan.stjude.cloud/proteinpaint). The total count of P/LPVs per exon (green) and intron (red) are depicted.

\section{Discussion}

In this study, we reported a patient with a personal and family history for FH. A tNGS-based approach allowed the identification of a previously unreported frameshift variant in the $L D L R$ gene. Although the functional consequence of this variant remains to be determined via in vitro functional assays, we can state that it can be considered as deleterious variant, according to the current variant classification guidelines.

This case confirmed the role of the $L D L R$ gene as main molecular target involved in $\mathrm{FH}$. 
P/LPVs in this gene can be divided into 6 classes affecting different aspects of LDL receptor function and based on their phenotypic behavior [17]. The class 2 comprises P/LPVs in which the normal routing through the cell is not completed or it is only very slowly completed. Usually, there is a complete blockade of transport, and LDL receptors are unable to leave the Endoplasmatic Reticulum (ER). Truncated proteins, as a result of a premature stop codon, and misfolded proteins are retained in the ER.

The c.171_184de/ variant falls in the exon 2 of the $L D L R$ gene in the LDL-receptor class A1 domain, causing a very premature stop codon. For these reasons, the protein is missing from all functional domains, resulting in a severe misfolded $L D L R$. Therefore, we can speculate that this variant belongs to class $2 \mathrm{~A}$ variants, commonly referred to a 'folding or conformational disease' [18], either unable to leave or leaving the ER for the Golgi at less than $5 \%$ [19].

The patient of this study is of Ukrainian origin. FH prevalence and features in this region are reported in two projects: the ScreenPro FH Project and the European Atherosclerosis Society FH Studies Collaboration $[12,13]$. The ScreenPro FH Project, an international project dedicated to the improvement of complex care-screening, diagnosis, and treatment of $\mathrm{FH}$ in the regions of Central, Eastern, and Southern Europe, estimated approximately $86,000 \mathrm{FH}$ in Ukraine (on a population of 43 million inhabitants) and only 147 patients have been included in the ScreenPro FH database. On the contrary, the EAS FHSC, an international initiative which aims to develop a worldwide, cross-regional registry of FH patients estimated about $160,000 \mathrm{FH}$ patients ( $~ 0.4 \%$ of the population). Currently, the EAS FHSC-related registry includes 165 Ukrainian patients.

This case report emphasizes how recent advances in genetic sequencing technology have resulted in remarkable improvements in speed, throughput and identification of P/LPVs occurring in FH patients. For these reasons, NGS is nowadays considered the main molecular technique for $\mathrm{FH}$ genetic diagnosis, with several NGS approaches to choose from: targeted panels for monogenic dyslipidemias [11, 20], whole exome sequencing, or extensive whole genome sequencing [21].

The progressive consolidation of NGS will have a strong impact on the knowledge of FH disease, allowing the development of public health approaches mainly aimed to early clinical FH diagnosis and treatment, especially in under-researched populations, such as the Ukrainian population, for which genetics of $\mathrm{FH}$ is still unexplored and poorly known.

\section{Conclusion}

This study showed that the integrated care model routinely adopted in our hospital for $\mathrm{FH}$ patients led to a discovery of a novel $L D L R P V$ in a Ukrainian family. A comprehensive model should include enrollment, specialist assessment, genetic testing and careful follow-up of $\mathrm{FH}$ patients. The expected benefits of the genetic testing in this family are: 1) unequivocal diagnosis of $\mathrm{FH}, 2$ ) planning of a personalized target therapy, 3) cascade family testing of the patient's relatives, and 4) a more accurate stratification of cardiovascular risk to prevent significant cardiovascular events. 
Finally, this case, in addition to expanding the knowledge of FH genetic basis in the Ukrainian population, confirmed that a tNGS strategy can be successfully used in the appropriate clinical setting.

\section{Abbreviations}

\section{$\mathrm{FH}$}

Familial Hypercholesterolemia; LDL-C:Low-Density Lipoprotein Cholesterol; DCLN:Dutch Lipid Clinic Network; LDLR:Low-Density Lipoprotein Receptor; APOB:Apolipoprotein B-100; PCSK9:Proprotein Convertase Subtilisin/Kexin type 9; LDLRAP1:Low-Density Lipoprotein Receptor Adaptor Protein 1; STAP1:Signal-Transducing Adaptor family member 1; NGS:Next Generation Sequencing; P/LPVs:Pathogenic/Likely Pathogenic Variants; tNGS:targeted NGS; EAS FHSC:European Atherosclerosis Society Familial Hypercholesterolemia Studies Collaboration; ESC:European Society of Cardiology; CNAs:Copy Number Alterations; VAF:Variant Allele Frequency; LOVD:Leiden Open-source Variation Database; ExAC:Exome Aggregation Consortium; HRMA:High Resolution Melting Analysis; ER:Endoplasmatic Reticulum.

\section{Declarations}

\section{Contributions}

SM and AM contributed conception and design of the study. MEO, EDP, CRT and AP performed the experiments and analyzed data. All authors contributed to manuscript revision, read, and approved the submitted version.

\section{Compliance with ethical standards}

\section{Conflict of interest}

The authors declared no potential conflicts of interest.

\section{Ethical approval}

This study complied with the Ethical Principles for Medical Research Involving Human Subjects according to the World Medical Association Declaration of Helsinki and was certified by the Committee of the Applicable Institution of the Fondazione Policlinico Universitario Agostino Gemelli IRCCS, Rome.

\section{Consent to participate}

The patient signed written informed consent before genetic tests.

\section{Consent to publish}

All authors have given their consent to participate in this report and submit it to Molecular Biology Reports 


\section{References}

1. Wilemon KA, Patel J, Aguilar-Salinas $C$ et al (2020) Reducing the clinical and public health burden of familial hypercholesterolemia: a global call to action. JAMA Cardiol 5:217-229. https://doi.org/10.1001/jamacardio.2019.5173

2. Nordestgaard BG, Chapman MJ, Humphries SE et al (2013) European Atherosclerosis Society Consensus Panel, Familial hypercholesterolaemia is underdiagnosed and undertreated in the general population: guidance for clinicians to prevent coronary heart disease: consensus statement of the European Atherosclerosis. Societ Eur Heart J 34:3478-3390a. https://doi.org/10.1093/eurheartj/eht273

3. Haralambos K, Ashfield-Watt P, McDowell IF (2016) Diagnostic scoring for familial hypercholesterolaemia in practice. Curr Opin Lipidol 27:367-374. https://doi.org/10.1097/MOL.0000000000000325

4. Defesche JC, Lansberg PJ, Umans-Eckenhausen MAW (2004) Advanced method for the identification of patients with inherited hypercholesterolemia. Semin Vasc Med 4:59-65. https://doi.org/10.1055/s-2004-822987

5. Betteridge DJ, Broome K, Durrington PN (1991) Risk of fatal coronary heart disease in familial hypercholesterolaemia. BMJ 303:893-896. https://doi.org/10.1136/bmj.303.6807.893

6. Craig IH (1995) Make early diagnosis, prevent early death from familial hypercholesterolaemia. The MED-PED FH program. Med J 162:454-455. https://doi.org/10.5694/j.1326-5377.1995.tb140001.x

7. Williams RR, Hunt SC, Schumacher MC et al (1993) Diagnosing heterozygous familial hypercholesterolemia using new practical criteria validated by molecular genetics. Am J Cardiol 72:171-176. https://doi.org/10.1016/0002-9149(93)90155-6

8. Sturm AC, Knowles JW, Gidding SS et al (2018) Convened by the Familial Hypercholesterolemia Foundation, Clinical Genetic Testing for Familial Hypercholesterolemia: JACC Scientific Expert Panel. Am J Cardiol 72:662-680. https://doi.org/10.1016/j.jacc.2018.05.044

9. Lui DTW, Lee ACH, Tan KCB (2020) Management of Familial Hypercholesterolemia: Current Status and Future Perspectives. J Endocr Soc 5:bvaa122. https://doi.org/10.1210/jendso/bvaa122

10. Henderson R, O'Kane M, McGilligan V et al (2016) The genetics and screening of familial hypercholesterolaemia. J Biomed Sci 23:39. https://doi.org/10.1186/s12929-016-0256-1

11. Moffa S, Mazzuccato G, De Bonis M et al (2020) Identification of two novel LDLR variants by Next Generation Sequencing. Ann Ist Super Sanita 56:122-127. https://doi.org/10.4415/ANN_20_01_17

12. Ceska R, Latkovskis G, Ezhov MV et al (2019) The Impact of the International Cooperation On Familial Hypercholesterolemia Screening and Treatment: Results from the ScreenPro FH Project. Curr Atheroscler Rep 21:36. doi.org/10.1007/s11883-019-0797-3

13. Vallejo-Vaz AJ, De Marco M, Stevens CAT et al (2018) Overview of the current status of familial hypercholesterolaemia care in over 60 countries - The EAS Familial Hypercholesterolaemia Studies 
Collaboration (FHSC). Investigators Atherosclerosis 277:234-255.

https://doi.org/10.1016/j.atherosclerosis.2018.08.051

14. Catapano AL, Chapman J, Wiklund $O$ et al (2011) The new joint EAS/ESC guidelines for the management of dyslipidaemias. Atherosclerosis 217:1.

https://doi.org/10.1016/j.atherosclerosis.2011.06.011

15. De Paolis E, Minucci A, De Bonis M et al (2018) A rapid screening of a recurrent CYP24A1 pathogenic variant opens the way to molecular testing for Idiopathic Infantile Hypercalcemia (IIH). Clin chim acta 482:8-13. https://doi.org/10.1016/j.cca.2018.03.024

16. Delibato E, Gattuso A, Minucci A et al (2009) PCR experion automated electrophoresis system to detect Listeria monocytogenes in foods. J Sep Sci 32:3817-3821. https://doi.org/10.1002/jssc.200900166

17. Benito-Vicente A, Uribe KB, Jebari S et al (2018) Validation of LDLr Activity as a Tool to Improve Genetic Diagnosis of Familial Hypercholesterolemia: A Retrospective on Functional Characterization of LDLr Variants. Int J Mol Sci 19:1676. https://doi.org/10.3390/ijms19061676

18. Gent J, Braakman I (2004) Low-density lipoprotein receptor structure and folding. Cell Mol Life Sci 61:2461-2470. https://doi.org/10.1007/s00018-004-4090-3

19. Hobbs HH, Russell DW, Brown MS et al (1990) The LDL receptor locus in familial hypercholesterolemia: mutational analysis of a membrane protein. Annu Rev Genet 24:133-170. https://doi.org/10.1146/annurev.ge.24.120190.001025

20. Reeskamp LF, Tromp TR, Defesche JC et al (2020) Next-generation sequencing to confirm clinical familial hypercholesterolemia. Eur J Prev Cardiol 27. https://doi.org/10.1177/2047487320942996

21. Khera AV, Chaffin M, Zekavat SM et al (2019) Whole-Genome Sequencing to Characterize Monogenic and Polygenic Contributions in Patients Hospitalized With Early-Onset Myocardial Infarction. Circulation 139:1593-1602. https://doi.org/10.1161/CIRCULATIONAHA.118.035658

\section{Tables}

Table 1 is not available with this version

\section{Figures}




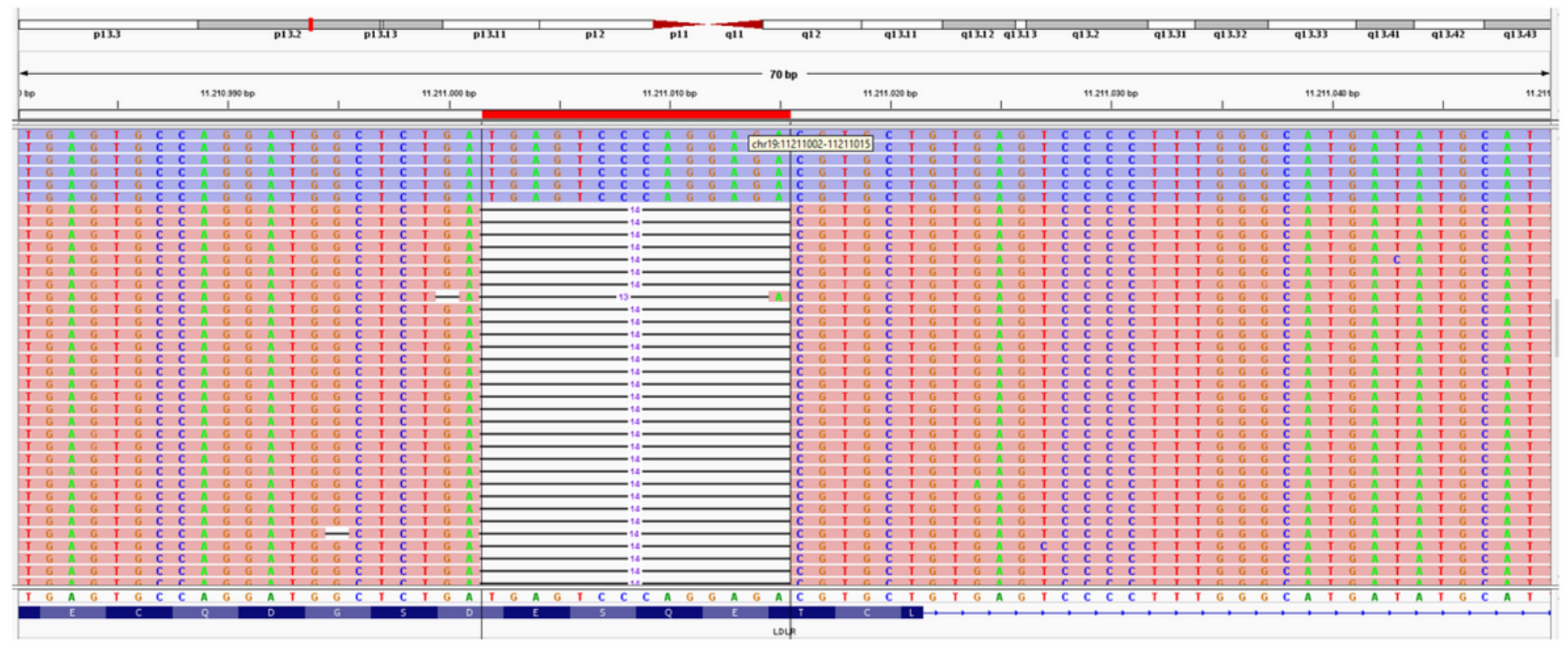

Figure 1

BAM files visualized by the Integrative Genomics Viewer show the reads associated to the c.171_184del, p.(Glu58ValfsTer67) variant with a coverage (variant/total calling) of 325/643X (variant allele frequency: $44 \%)$. 
A)

; $C T G A G T G C C A G G A T G G C T C T G A Y G W G Y Y S Y G A G W C A C C T T T G G K S$

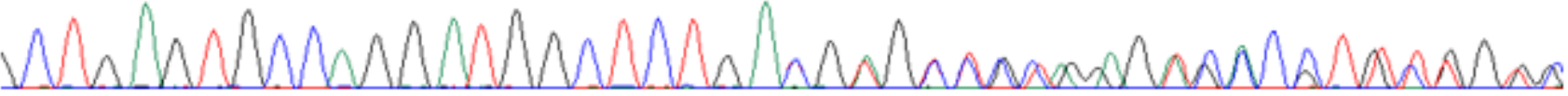

B)

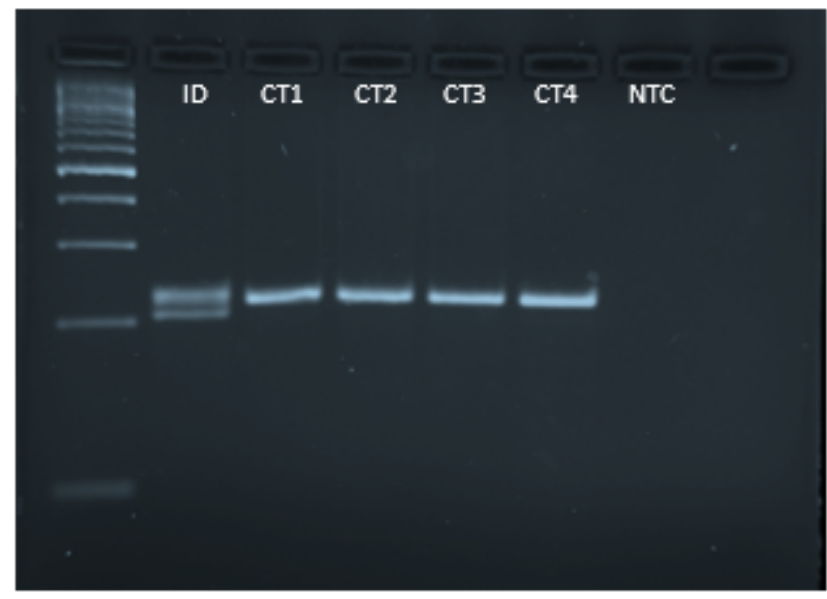

C)
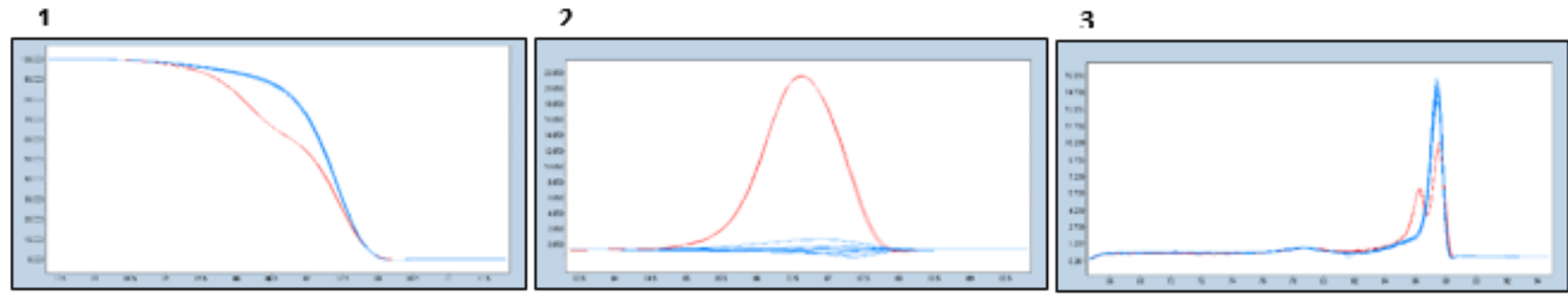

\section{Figure 2}

This figure shows the results of different molecular approaches used to confirm the LDLR c.171_184del variant. A) Sanger Sequencing: the presence of the variant is demonstrated with a double sequence caused by the non-in frame deletion (Applied Biosystems 3500 Genetic Analyzer -Life Technologies, Carlsbad, CA, USA- and SeqScape Software v2.5); B) DNA agarose gel electrophoresis shows the presence of a double band in the patient's PCR product (ID) compared to controls (CT) with a single band. DNA agarose gel electrophoresis was performed for $45 \mathrm{~min}$ at $100 \mathrm{~V}$ with $4 \%$ agarose gel in a buffer containing $89 \mathrm{mM}$ Tris, $89 \mathrm{mM}$ borate, and $2 \mathrm{mM}$ EDTA (TBE) at a final concentration of 1X. The DNA quantification was performed via a $100 \mathrm{bp}$ marker (Thermo Fisher Scientific) at $0.5 \mu \mathrm{g} / \mathrm{ul}$ concentration. PCR products were revealed by measuring ethidium bromide chemifluorescence with ChemiDoc XRS+ System (Bio-Rad); C) High Resolution Melting Analysis (HRMA) of LDLR c.171_184del variant. The three panels show: Normalized and Temperature-shifted Plot (1), Difference Plot (2) and Derivative Plot (3), 
respectively. Data were reported for wild-type (blue) and patient samples (red) sample. Patient's panels show different melting profiles compared to control.

\begin{tabular}{ccccccc}
\hline $\begin{array}{c}\text { Number of } \\
\text { LDLR P/LPVs }\end{array}$ & Nonsense & Missense & Indels & Cna & Splicing & Synonymus \\
\hline $\mathbf{1 4 5 2}$ & 127 & 644 & 418 & 111 & 132 & 20 \\
\hline
\end{tabular}

\section{Figure 3}

This figure shows the total LDLR P / LPV from the UCL LOVD database reported by variant type

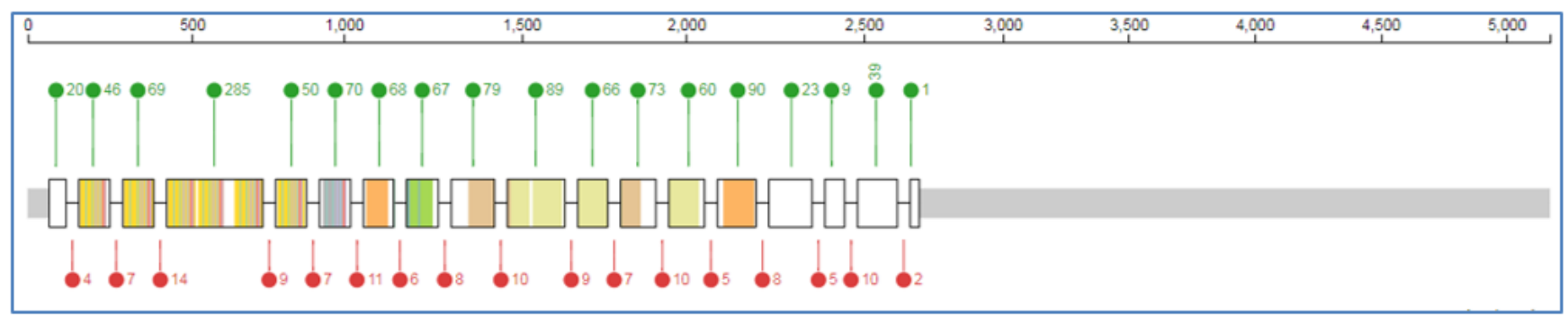

\section{Figure 4}

The figure shows the linear map of the low-density lipoprotein receptor (LDLR) gene (NM_000527) with the total number of the P/LPVs accounted in each exon (green) and intron (red). 